\title{
Chebyshev diagrams for two-bridge knots
}

\author{
P. - V. Koseleff, D. Pecker \\ Université Pierre et Marie Curie \\ 4, place Jussieu, F-75252 Paris Cedex 05 \\ e-mail: \{koseleff, pecker\}@math.jussieu.fr
}

November 8, 2018

\begin{abstract}
We show that every two-bridge knot $K$ of crossing number $N$ admits a polynomial parametrization $x=T_{3}(t), y=T_{b}(t), z=C(t)$ where $T_{k}(t)$ are the Chebyshev polynomials and $b+\operatorname{deg} C=3 N$. If $C(t)=T_{c}(t)$ is a Chebyshev polynomial, we call such a knot a harmonic knot. We give the classification of harmonic knots for $a \leq 3$. Most results are derived from continued fractions and their matrix representations.
\end{abstract}

keywords: Polynomial curves, Chebyshev polynomials, Chebyshev curves, rational knots, continued fractions

Mathematics Subject Classification 2000: 14H50, 57M25, 14P99

\section{Contents}

1 Introduction 2

2 Continued fractions and rational Chebyshev knots 4

3 Continued fractions $[ \pm \mathbf{1}, \pm \mathbf{1}, \ldots, \pm \mathbf{1}] \quad \mathbf{7}$

4 Chebyshev diagrams of rational knots 12

5 Polynomial parametrization of rational knots 14

6 The harmonic knots $\mathrm{H}(3, \mathrm{~b}, \mathrm{c}) \quad 17$

7 Proof of theorem 6.5 21

8 Conclusion 23 


\section{Introduction}

We study the polynomial parametrization of knots, viewed as non singular space curves. Vassiliev proved that any knot can be represented by a polynomial embedding $\mathbf{R} \rightarrow \mathbf{R}^{3} \subset \mathbf{S}_{3}$ ([Va] $)$. Shastri ([Sh] $)$ gave another proof of this theorem, he also found explicit parametrizations of the trefoil and of the figure-eight knot.

We shall study polynomial embeddings of the form $x=T_{a}(t), y=T_{b}(t), z=C(t)$ where $a$ and $b$ are coprime integers and $T_{n}(t)$ are the classical Chebyshev polynomials defined by $T_{n}(\cos t)=\cos n t$. The projection of such a curve on the $x y$-plane is the Chebyshev curve $\mathcal{C}(a, b): T_{b}(x)=T_{a}(y)$ which has exactly $\frac{1}{2}(a-1)(b-1)$ crossing points ([Fi, [P1]). We will say that such a knot has the Chebyshev diagram $\mathcal{C}(a, b)$.

We observed in KP1 that the trefoil can be parametrized by Chebyshev polynomials: $x=T_{3}(t) ; y=T_{4}(t) ; z=T_{5}(t)$. This led us to study Chebyshev knots in [KP3. We obtained the following result:

Theorem 1.1 ([KP3]). Any knot is a Chebyshev knot, that is, is isotopic to a knot given by a one-to-one parametrization

$$
\mathcal{C}(a, b, c, \varphi): x=T_{a}(t) ; y=T_{b}(t) ; z=T_{c}(t+\varphi)
$$

where $t \in \mathbf{R}, a$ and $b$ are coprime integers, $c$ is an integer and $\varphi$ is a real constant.

Our proof uses theorems on braids by Hoste, Zirbel and Lamm ([HZ, La2]), and a density argument. In a joint work with F. Rouillier ([KPR $)$, we developed an effective method to enumerate all the knots $\mathcal{C}(a, b, c, \varphi), \varphi \in \mathbf{R}$ where $a=3$ or $a=4, a$ and $b$ coprime.

Chebyshev knots are polynomial analogues of Lissajous knots that admit a parametrization of the form

$$
x=\cos (a t) ; y=\cos (b t+\varphi) ; z=\cos (c t+\psi)
$$

where $0 \leq t \leq 2 \pi$ and where $a, b, c$ are pairwise coprime integers. These knots, introduced in [BHJS, have been studied by V. F. R. Jones, J. Przytycki, C. Lamm, J. Hoste and L. Zirbel. Most known properties of Lissajous knots are deduced from their symmetries (see [BDHZ, Cr, HZ, JP, La1]).

Definition 1.2. When $a, b, c$ are coprime then $\mathcal{C}(a, b, c, 0)$ is denoted by $\mathrm{H}(a, b, c)$ and is called a harmonic knot.

The symmetries of harmonic knots, obvious from the parity of Chebyshev polynomials, are different from those of Lissajous. For example, the figure-eight knot which is amphicheiral but not a Lissajous knot, is the harmonic knot $\mathrm{H}(3,5,7)$.

We proved in $\mathrm{KP}$ ] that the harmonic knot $\mathrm{H}(a, b, a b-a-b)$ is alternating, and deduced that there are infinitely many amphicheiral harmonic knots and infinitely many strongly 
invertible harmonic knots. We also proved in [KP3] that the torus knot $\mathrm{T}(2,2 n+1)$ is the harmonic knot $\mathrm{H}(3,3 n+2,3 n+1)$.

In this article, we give the classification of the harmonic knots $\mathrm{H}(a, b, c)$ for $a \leq 3$. We also give explicit polynomial parametrizations of all rational knots. The diagrams of our knots are Chebyshev curves of minimal degrees with a small number of crossing points. The degrees of the height polynomials are small.

In section 2 we recall the Conway notation for rational knots, and the computation of their Schubert fractions with continued fractions. We observe that, when $a=3$, Chebyshev diagrams correspond to continued fractions of the form $[ \pm 1, \ldots, \pm 1]$.

The study of these particular continued fraction expansions will be the main tool of this article.

\section{Theorem 3.1.}

Every rational number $r$ has a unique continued fraction expansion $r=\left[e_{1}, e_{2}, \ldots, e_{n}\right]$, $e_{i}= \pm 1$, where there are no two consecutive sign changes in the sequence $\left(e_{1}, \ldots, e_{n}\right)$.

We provide a formula (Proposition 2.5) for the crossing number of the corresponding knots. Then we study the matrix interpretation of these continued fraction expansions.

In section 4 we show how to find explicit minimal Chebyshev diagrams $\mathcal{C}(3, b)$ for all rational knots:

\section{Theorem 4.3 .}

Let $K$ be a two-bridge knot with crossing number $N$. There is an algorithm to determine the smallest $b$ such that $K$ has a Chebyshev diagram $\mathcal{C}(3, b)$ with $N<b<\frac{3}{2} N$.

As an application, we give optimal Chebyshev diagrams for the torus knots $\mathrm{T}(2, N)$, the twist knots $\mathcal{T}_{n}$, the generalized stevedore knots and some others.

In section 5, we find explicit polynomial parametrizations of all rational knots. We first use the minimal Chebyshev diagram found in section 4, then we define a height polynomial of small degree. More precisely, we show:

\section{Theorem 5.2,}

Every rational knot of crossing number $N$ can be parametrized by $x=T_{3}(t), y=T_{b}(t), z=$ $C(t)$ where $b+\operatorname{deg} C=3 N$. Furthermore, when the knot is amphicheiral, $b$ is odd and we can choose $C$ to be an odd polynomial.

We give the first polynomial parametrizations of the twist knots and the generalized stevedore knots.

In section 6, we give a complete classification of harmonic knots $\mathrm{H}(3, b, c)$ where $b$ and $c$ are relatively prime integers, not divisible by 3 . We obtain this classification by a careful study of the diagrams and their continued fractions (Theorem 6.5 whose proof is given in section (7). We show that the twist knots and the generalized stevedore knots (e.g. the $6_{1}$ knot) are not harmonic knots $\mathrm{H}(3, b, c)$. 
Thanks to the use of continued fractions, we provide effective methods for the construction of polynomial parametrizations for any rational knot. We conjecture they are of minimal degrees.

\section{Continued fractions and rational Chebyshev knots}

A two-bridge knot (or link) admits a diagram in Conway's normal form. This form, denoted by $C\left(a_{1}, a_{2}, \ldots, a_{n}\right)$ where $a_{i}$ are integers, is explained by the following picture (see [Con], [Mu p. 187). The number of twists is denoted by the integer $\left|a_{i}\right|$, and the sign of $a_{i}$ is
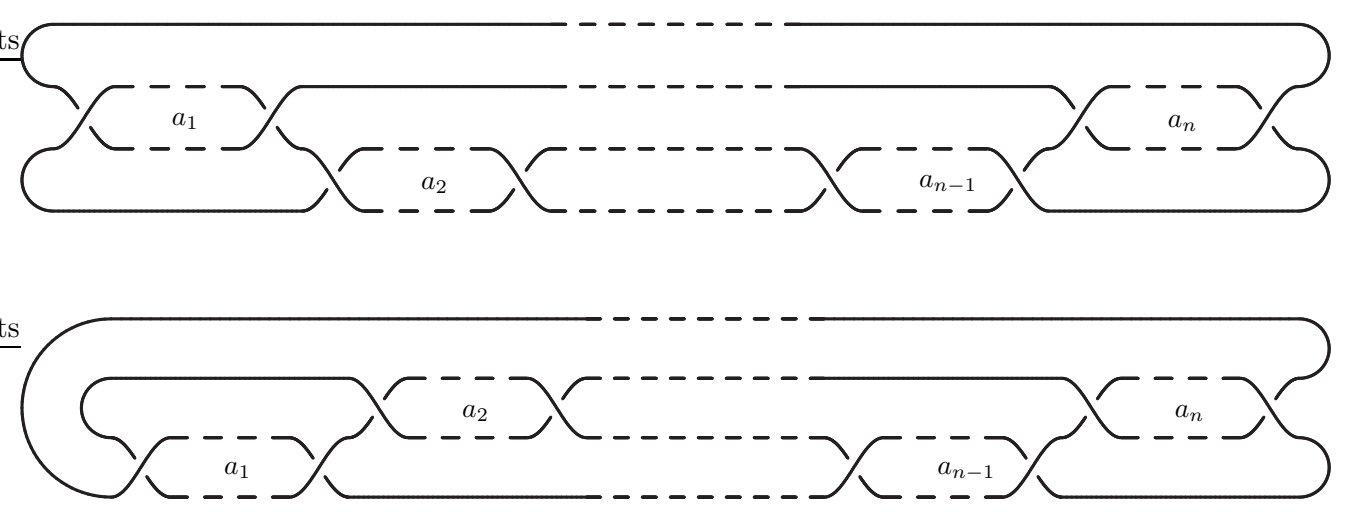

Figure 1: Conway's normal forms, $n$ odd, $n$ even

defined as follows: if $i$ is odd, then the right twist is positive, if $i$ is even, then the right twist is negative. On Fig. 1 the $a_{i}$ are positive (the $a_{1}$ first twists are right twists).

Examples 2.1. The trefoil has the following Conway's normal forms $C(3), C(-1,-1,-1)$, $C(4,-1)$ and $C(-1,-1,1,1)$. The diagrams in Figure 2 clearly represent the same trefoil.

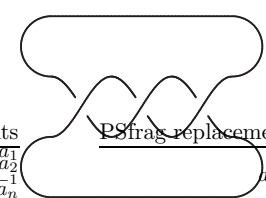

$C(3)$

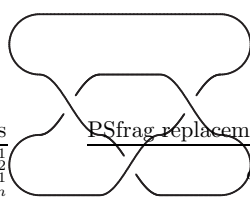

$C(-1,-1,-1)$

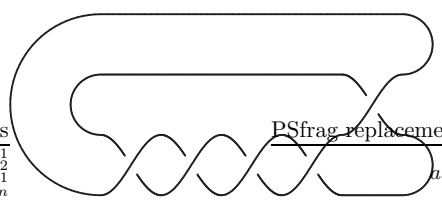

$C(4,-1)$

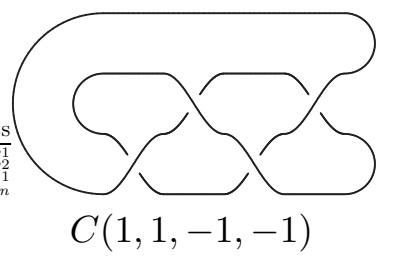

Figure 2: Diagrams of the standard trefoil 
The two-bridge links are classified by their Schubert fractions

$$
\frac{\alpha}{\beta}=a_{1}+\frac{1}{a_{2}+\frac{1}{a_{3}+\frac{1}{\cdots+\frac{1}{a_{n}}}}}=\left[a_{1}, \ldots, a_{n}\right], \quad \alpha>0
$$

We shall denote by $S\left(\frac{\alpha}{\beta}\right)$ a two-bridge link with Schubert fraction $\frac{\alpha}{\beta}$. The two-bridge links $S\left(\frac{\alpha}{\beta}\right)$ and $S\left(\frac{\alpha^{\prime}}{\beta^{\prime}}\right)$ are equivalent if and only if $\alpha=\alpha^{\prime}$ and $\beta^{\prime} \equiv \beta^{ \pm 1}(\bmod \alpha)$. The integer $\alpha$ is odd for a knot, and even for a two-component link. If $K=S\left(\frac{\alpha}{\beta}\right)$, its mirror image is $\bar{K}=S\left(\frac{\alpha}{-\beta}\right)$.

We shall study knots with a Chebyshev diagram $\mathcal{C}(3, b): x=T_{3}(t), y=T_{b}(t)$. It is remarkable that such a diagram is already in Conway normal form: the crossing points belong to the 2 horizontal lines $y= \pm \frac{1}{2} \sqrt{3}$. Consequently, the Schubert fraction of such a knot is given by a continued fraction of the form $[ \pm 1, \pm 1, \ldots, \pm 1]$. For example the only diagrams of Figure 2 which may be Chebyshev are the second and the last (in fact they are Chebyshev).

Figure 3 shows a typical example of a knot with a Chebyshev diagram.

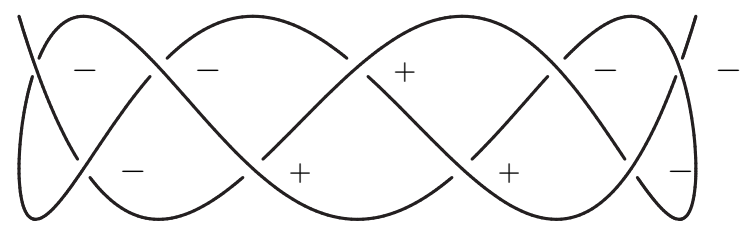

Figure 3: A Chebyshev diagram of the torus knot $\mathrm{T}(2,7)$

This knot is defined by $x=T_{3}(t), y=T_{10}(t), z=-T_{11}(t)$. Its $x y$-projection is in the Conway normal form $C(-1,-1,-1,1,1,1,-1,-1,-1)$. Its Schubert fraction is then $\frac{7}{-6}$ and this knot is the torus knot $\mathrm{T}(2,7)=S\left(\frac{7}{-6}\right)=S(7)$.

Let $\alpha, \beta$ be relatively prime integers. Then $\frac{\alpha}{\beta}$ admits the continued fraction expansion 
$\frac{\alpha}{\beta}=\left[q_{1}, q_{2}, \ldots, q_{n}\right]$ if and only if there exist integers $r_{i} \in \mathbf{Z}$ such that

$$
\left\{\begin{aligned}
\alpha & =q_{1} \beta+r_{2}, \\
\beta & =q_{2} r_{2}+r_{3} \\
& \vdots \\
r_{n-2} & =q_{n-1} r_{n-1}+r_{n} \\
r_{n-1} & =q_{n} r_{n} .
\end{aligned}\right.
$$

The integers $q_{i} \in \mathbf{Z}$ are called the quotients of the continued fraction. Euclidean algorithms provide various continued fraction expansions which are useful to the study of two-bridge knots (see [BZ, $[\mathrm{St}, \mathrm{Cr}]$ ).

If $\alpha>\beta>0$, there is a unique continued expansion of $\frac{\alpha}{\beta}=\left[q_{1}, \ldots, q_{n}\right], q_{i}>0$ up to $q_{n}=\left[q_{n}-1,1\right]$.

Definition 2.2. Let $r>1$ be a rational number, and $r=\left[q_{1}, \ldots, q_{n}\right]$ be its classical continued fraction expansion (with $q_{i}>0$ ). The crossing number of $r$ is defined by $\mathrm{cn}(r)=$ $q_{1}+\cdots+q_{n}$

Remark 2.3. One can prove that $\operatorname{cn}\left(\frac{\alpha}{\beta}\right)=\operatorname{cn}\left(\frac{\alpha}{\alpha-\beta}\right)$ when $\frac{\alpha}{\beta}>1$. If $K=S\left(\frac{\alpha}{\beta}\right), \frac{\alpha}{\beta}>1$ is a rational knot, it is known that $\operatorname{cn}\left(\frac{\alpha}{\beta}\right)$ is the crossing number of $K$. It means that it is the minimum number of crossing points for all diagrams of $K([\underline{\mathrm{Mu}}])$.

We shall be interested in algorithms where the sequence of remainders is not necessarily decreasing anymore (the $q_{i}$ are not necessarily positive).

Definition 2.4. A continued fraction $\left[a_{1}, a_{2}, \ldots, a_{n}\right]$ is 1-regular if it has the following properties:

$$
a_{i} \neq 0, a_{n-1} a_{n}>0, \text { and } a_{i} a_{i+1}<0 \Rightarrow a_{i+1} a_{i+2}>0, i=1, \ldots, n-2 .
$$

Proposition 2.5. Let $\frac{\alpha}{\beta}=\left[a_{1}, \ldots, a_{n}\right]$ be a 1-regular continued fraction with $a_{1}, a_{2}>0$. Then $\frac{\alpha}{\beta}>1$ and we have

$$
\operatorname{cn}\left(\frac{\alpha}{\beta}\right)=\sum_{k=1}^{n}\left|a_{i}\right|-\sharp\left\{i, a_{i} a_{i+1}<0\right\} .
$$

Proof. We prove this result by induction on the number of sign changes $k=\sharp\left\{i, a_{i} a_{i+1}<0\right\}$. If $k$ is 0 , it is the definition. If $k>0$ let us consider the first change of sign. The 1-regular continued fraction is $[x, a, b,-c,-d,-y]$ where $a, b, c, d$ are positive integers, $x$ is a sequence (possibly empty) of positive integers and $y$ is a 1-regular sequence of integers. We have $[x, a, b,-c,-d,-y]=[x, a, b-1,1, c-1, d, y]$ (Lagrange identity, $[\mathrm{Cr}])$ 
- Suppose $(b-1)(c-1)>0$, then the sum of absolute values has decreased by 1 and the number of changes of sign has also decreased by 1 .

- Suppose $b=1, c \neq 1$ (resp. $c=1, b \neq 1$ ). Then $[x, a, b,-c,-d,-y]=[x, a, 0,1, c-$ $1, d, y]=[x, a+1, c-1, d, y]$. (resp. $[x, a-1, c+1, d, y]$ ). The sum of absolute values has decreased by 1 and the number of changes of sign has also decreased by 1 .

- Suppose $b=c=1$. Then $[x, a, b,-c,-d,-y]=[x, a, 0,1,0, d, y]=[x, a+d+1, y]$. The sum of absolute values has decreased by 1 and the number of changes of sign has also decreased by 1 .

We therefore deduce that $\frac{\alpha}{\beta}=[x, r]$ where $r>1$ and $x$ is a sequence sequence (possibly empty) of positive integers. We get $\frac{\alpha}{\beta}>1$.

Note that Formula (11) still holds when $a_{1}, \ldots, a_{n}$ are non zero even integers and the sequence is not necessarily 1-regular (see $[\mathrm{St}]$ ).

We shall now use the basic (subtractive) Euclidean algorithm to get 1-regular continued fractions of the form $[ \pm 1, \pm 1, \ldots, \pm 1]$.

\section{Continued fractions $[ \pm 1, \pm \mathbf{1}, \ldots, \pm \mathbf{1}]$}

We will consider the following Möbius transformations:

$$
P: x \mapsto[1, x]=1+\frac{1}{x}, M: x \mapsto[1,-1,-x]=\frac{1}{1+x} .
$$

Let $E$ be the set of positive real numbers. We have $P(E)=] 1, \infty[$ and $M(E)=] 0,1[. P(E)$ and $M(E)$ are disjoint subsets of $E$.

Theorem 3.1. Let $\frac{\alpha}{\beta}$ be a rational number. There is a unique 1-regular continued fraction such that

$$
\frac{\alpha}{\beta}=\left[e_{1}, e_{2}, \ldots, e_{n}\right], e_{i}= \pm 1
$$

Furthermore, $\frac{\alpha}{\beta}>1$ if and only if $e_{1}=e_{2}=1$.

Proof. Let us prove the existence by induction on the height $h\left(\frac{\alpha}{\beta}\right)=\alpha+\beta$.

- If $h=2$ then $\frac{\alpha}{\beta}=1=[1]$ and the result is true.

- If $\alpha>\beta$, we have $\frac{\alpha}{\beta}=P\left(\frac{\beta}{\alpha-\beta}\right)=\left[1, \frac{\beta}{\alpha-\beta}\right]$. Since $h\left(\frac{\beta}{\alpha-\beta}\right)<h\left(\frac{\alpha}{\beta}\right)$, we get our 1-regular continued fraction for $r$ by induction. 
- If $\beta>\alpha$ we have $\frac{\alpha}{\beta}=M\left(\frac{\beta-\alpha}{\alpha}\right)=\left[1,-1,-\frac{\beta-\alpha}{\alpha}\right]$. And we also get a 1-regular continued fraction for $r$.

This completes the construction of our continued fraction expansion.

On the other hand, let $r$ be defined by the 1-regular continued fraction $r=\left[1, r_{2}, \ldots, r_{n}\right]$, $r_{i}= \pm 1, n \geq 2$. Let us prove, by induction on the length $n$ of the continued fraction, that $r>0$ and that $r>1$ if and only if $r_{2}=1$.

- If $r_{2}=1$ we have $r=P\left(\left[1, r_{3}, \ldots, r_{n}\right]\right)$, and by induction $r \in P(E)$ and then $r>1$.

- If $r_{2}=-1$, we have $r_{3}=-1$ and $r=M\left(\left[1,-r_{4}, \ldots,-r_{n}\right]\right)$. By induction, $r \in$ $M(E)=] 0,1[$.

The uniqueness is now easy to prove. Let $r=\left[1, r_{2}, \ldots, r_{n}\right]=\left[1, r_{2}^{\prime}, \ldots, r_{n^{\prime}}^{\prime}\right]$.

- If $r>1$ then $r_{2}=r_{2}^{\prime}=1$ and $\left[1,1, r_{3}, \ldots, r_{n}\right]=\left[1,1, r_{3}^{\prime}, \ldots r_{n^{\prime}}^{\prime}\right]$. Consequently, $\left[1, r_{3}, \ldots, r_{n}\right]=\left[1, r_{3}^{\prime}, \ldots, r_{n^{\prime}}^{\prime}\right]$, and by induction $r_{i}=r_{i}^{\prime}$ for all $i$.

- If $r<1$, then $r_{2}=r_{3}=r_{2}^{\prime}=r_{3}^{\prime}=-1$ and $\left[1,-1,-1, r_{4}, \ldots, r_{n}\right]=\left[1,-1,-1, r_{4}^{\prime}, \ldots, r_{n^{\prime}}^{\prime}\right]$. Then, $\left[1,-r_{4}, \ldots,-r_{n}\right]=\left[1,-r_{4}^{\prime}, \ldots,-r_{n^{\prime}}^{\prime}\right]$ and by induction $r_{i}=r_{i}^{\prime}$ for all $i$.

Remark 3.2. Since we have $[1,-1,1, x]=-x$, we see that 1-regularity is required for the uniqueness of our expansion. This is exactly analogous to the classical case.

Definition 3.3. Let $\frac{\alpha}{\beta}>0$ be the 1-regular continued fraction $\left[e_{1}, \ldots, e_{n}\right], e_{i}= \pm 1$. We will denote its length $n$ by $\ell\left(\frac{\alpha}{\beta}\right)$. Note that $\ell\left(-\frac{\alpha}{\beta}\right)=\ell\left(\frac{\alpha}{\beta}\right)$.

Examples 3.4. Using our algorithm we obtain

$$
\begin{aligned}
\frac{9}{7} & =\left[1, \frac{7}{2}\right]=\left[1,1, \frac{2}{5}\right]=\left[1,1,1,-1,-\frac{3}{2}\right]=\left[1,1,1,-1,-1,-\frac{2}{1}\right]=[1,1,1,-1,-1,-1,-1], \\
\frac{9}{2} & =\left[1, \frac{2}{7}\right]=\left[1,1,-1,-\frac{5}{2}\right]=\left[1,1,-1,-1,-\frac{2}{3}\right]=\left[1,1,-1,-1,-1,1, \frac{1}{2}\right] \\
& =[1,1,-1,-1,-1,1,1,-1,-1]=[4,2] .
\end{aligned}
$$

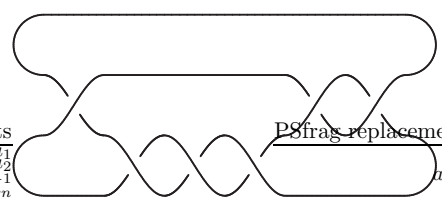

$C(1,3,2)$

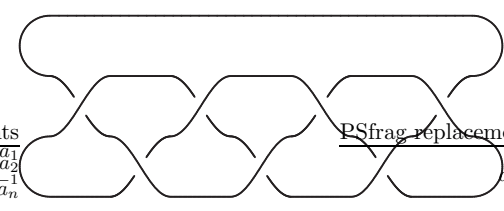

$C(1,1,1,-1,-1,-1,-1)$

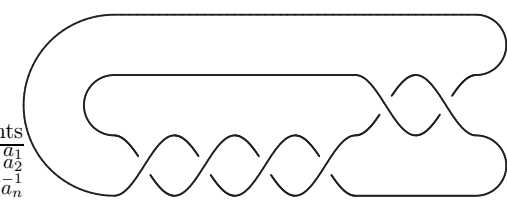

$C(4,2)$

Figure 4: Diagrams of the knot $6_{1}=S\left(\frac{9}{7}\right)$ and its mirror image $S\left(\frac{9}{2}\right)$ 
We will rather use the notation

$$
\frac{9}{7}=P^{2} M P^{3}(\infty), \frac{9}{2}=P M P M^{2} P(\infty) .
$$

We get $\ell\left(\frac{9}{7}\right)=7, \ell\left(\frac{9}{2}\right)=9$. The crossing numbers of these fractions are cn $\left(\frac{9}{7}\right)=$ $\operatorname{cn}([1,3,2])=6=7-1$ and $\operatorname{cn}\left(\frac{9}{2}\right)=\operatorname{cn}([4,2])=6=9-3$. If the fractions $\frac{9}{7}$ and $\frac{9}{2}$ have the same crossing number, it is because the knot $S\left(\frac{9}{7}\right)$ is the mirror image of $S\left(\frac{9}{2}\right)$. In order to get a full description of two-bridge knots we shall need a more detailed study of the homographies $P$ and $M$.

Proposition 3.5. The multiplicative monoid $\mathbf{G}=\langle P, M\rangle$ is free. The mapping $g: G \mapsto$ $G(\infty)$ is a bijection from $\mathbf{G} \cdot P$ to $\mathbf{Q}_{>0}$ and $g(P \cdot \mathbf{G} \cdot P)=\mathbf{Q}_{>1}$, the set of rational numbers greater than 1.

Proof. Suppose that $P X=M X^{\prime}$ for some $X, X^{\prime}$ in G. Then we would have $P X(1)=$ $M X^{\prime}(1) \in P(E) \bigcap M(E)=\emptyset$. Clearly, this implies that $\mathbf{G}$ is free. Similarly, from $P(\infty)=$ 1 , we deduce that the mapping $G \mapsto G \cdot P(\infty)$ is injective. From Theorem 3.1 and $P(\infty)=1$, we deduce that $g$ is surjective.

Remark 3.6. Let $r=G(\infty)=\left[e_{1}, \ldots, e_{n}\right], e_{i}= \pm 1$, be a 1-regular continued fraction. It is easy to find the unique homography $G \in \mathbf{G} \cdot P$ such that $r=G(\infty)$. Consider the sequence $\left(e_{1}, \ldots, e_{n}\right)$. For any $i$ such that $e_{i} e_{i+1}<0$, replace the couple $\left(e_{i}, e_{i+1}\right)$ by $M$, and then replace each remaining $e_{i}$ by $P$.

Let $G=P^{p_{1}} M^{m_{1}} \cdots M^{m_{k}} P^{p_{k+1}}$. Let $p=p_{1}+\cdots+p_{k+1}$ be the degree of $G$ in $P$ and $m=m_{1}+\cdots+m_{k}$ its degree in $M$. Then we have $n=\ell(r)=p+2 m$ and $\mathrm{cn}(r)=p+m$.

We shall consider matrix notations for many proofs. We will consider

$$
\left[\begin{array}{l}
\alpha \\
\beta
\end{array}\right]=P^{p_{1}} M^{m_{1}} \cdots M^{m_{k}} P^{p_{k+1}}\left[\begin{array}{l}
1 \\
0
\end{array}\right], \quad P=\left[\begin{array}{ll}
1 & 1 \\
1 & 0
\end{array}\right], \quad M=\left[\begin{array}{ll}
0 & 1 \\
1 & 1
\end{array}\right] .
$$

\section{Definition 3.7.}

We define on $\mathbf{G}$ the anti-homomorphism $G \mapsto \bar{G}$ by $\bar{M}=M, \bar{P}=P$.

We define on $\mathbf{G}$ the homomorphism $G \mapsto \widehat{G}$ by $\widehat{M}=P, \widehat{P}=M$.

Proposition 3.8. Let $\alpha>\beta>0$ and consider $\frac{\alpha}{\beta}=P G P(\infty)$ and $N=\operatorname{cn}\left(\frac{\alpha}{\beta}\right)$. Let $\beta^{\prime}$ be such that $0<\beta^{\prime}<\alpha$ and $\beta \beta^{\prime} \equiv(-1)^{N-1}(\bmod \alpha)$. Then we have

$$
\frac{\beta}{\alpha}=M \widehat{G} P(\infty), \quad \frac{\alpha}{\alpha-\beta}=P \widehat{G} P(\infty), \quad \frac{\alpha}{\beta^{\prime}}=P \bar{G} P(\infty)
$$


We also have

$$
\ell\left(\frac{\beta}{\alpha}\right)+\ell\left(\frac{\alpha}{\beta}\right)=3 N-1, \quad \ell\left(\frac{\alpha}{\alpha-\beta}\right)+\ell\left(\frac{\alpha}{\beta}\right)=3 N-2, \quad \ell\left(\frac{\alpha}{\beta^{\prime}}\right)=\ell\left(\frac{\alpha}{\beta}\right) .
$$

Proof. We use matrix notations for this proof. Let us consider $P G P=\left[\begin{array}{ll}\alpha & \beta^{\prime} \\ \beta & \alpha^{\prime}\end{array}\right]=$ $P^{p_{1}} M^{m_{1}} \cdots M^{m_{k}} P^{p_{k+1}}$. From $\operatorname{det} P=\operatorname{det} M=-1$, we obtain $\alpha \alpha^{\prime}-\beta \beta^{\prime}=(-1)^{N}$. Let $A=\left[\begin{array}{ll}a & c \\ b & d\end{array}\right]$ be a matrix such that $0 \leq c \leq a$ and $0 \leq d \leq b$. From $P A=\left[\begin{array}{cc}a+c & b+d \\ a & c\end{array}\right]$ and $M A=\left[\begin{array}{cc}b & d \\ a+b & c+d\end{array}\right]$ we deduce that $P G P$ satisfies $0<\alpha^{\prime}<\beta$ and $0<\beta^{\prime}<\alpha$. We therefore conclude that, $\beta^{\prime}$ is the integer defined by $0<\beta^{\prime}<\alpha, \beta \beta^{\prime} \equiv(-1)^{N-1}(\bmod \alpha)$. By transposition we deduce that

$$
\left[\begin{array}{cc}
\alpha & \beta \\
\beta^{\prime} & \alpha^{\prime}
\end{array}\right]=P^{p_{k+1}} M^{m_{k}} \cdots M^{m_{1}} P^{p_{1}}=P \bar{G} P
$$

which implies $\frac{\alpha}{\beta^{\prime}}=P \bar{G} P(\infty)$.

Let us introduce $J=\left[\begin{array}{ll}0 & 1 \\ 1 & 0\end{array}\right]$. We have $J^{2}=\mathbf{I d}, M=J P J$ and $P=J M J$. Therefore

$$
\left[\begin{array}{l}
\beta \\
\alpha
\end{array}\right]=J\left[\begin{array}{l}
\alpha \\
\beta
\end{array}\right]=M^{p_{1}} P^{m_{1}} \ldots P^{m_{k}} M^{p_{k+1}-1} J P\left[\begin{array}{l}
1 \\
0
\end{array}\right]=M^{p_{1}} P^{m_{1}} \cdots P^{m_{k}} M^{p_{k+1}-1} P\left[\begin{array}{l}
1 \\
0
\end{array}\right]
$$

that is $\frac{\beta}{\alpha}=M \widehat{G} P(\infty)$.

Then, $\left[\begin{array}{c}\alpha \\ \alpha-\beta\end{array}\right]=P M^{-1}\left[\begin{array}{l}\beta \\ \alpha\end{array}\right]=P \widehat{G} P\left[\begin{array}{l}1 \\ 0\end{array}\right]$. That is $\frac{\alpha}{\alpha-\beta}=P \widehat{G} P(\infty)$.

Relations on lengths are derived from the previous relations and remark 3.6.

Remark 3.9. It is straightforward that if $\left[\varepsilon_{1}, \ldots, \varepsilon_{n}\right]=P G P(\infty)$ then $P \bar{G} P(\infty)=$ $\varepsilon_{n}\left[\varepsilon_{n}, \ldots, \varepsilon_{1}\right]$. We deduce from Proposition 2.5 that $\operatorname{cn}\left(\frac{\alpha}{\beta}\right)=\operatorname{cn}\left(\frac{\alpha}{\beta^{\prime}}\right)$, as expected.

Lemma 3.10. Let $\frac{\alpha}{\beta}=\left[e_{1}, \ldots, e_{n}\right]$ be a 1-regular continued fraction $\left(e_{i}= \pm 1\right)$. We have

- $n \equiv 2(\bmod 3)$ if and only if $\alpha$ is even and $\beta$ is odd.

- $n \equiv 0(\bmod 3)$ if and only if $\alpha$ is odd and $\beta$ is even.

- $n \equiv 1(\bmod 3)$ if and only if $\alpha$ and $\beta$ are odd.

Proof. Let us write $\left[\begin{array}{l}\alpha \\ \beta\end{array}\right]=P^{p_{1}} M^{m_{1}} \cdots M^{m_{k}} P^{p_{k+1}}\left[\begin{array}{l}1 \\ 0\end{array}\right]$. Since $M \equiv P^{2}(\bmod 2)$, and $n=p+2 m$, we get $P^{p_{1}} M^{m_{1}} \cdots M^{m_{k}} P^{p_{k+1}} \equiv P^{n}(\bmod 2)$. As $P^{3} \equiv \mathbf{I d}(\bmod 2)$ we obtain 


$$
\begin{aligned}
& \text { if } n \equiv 2(\bmod 3) \text {, then }\left[\begin{array}{l}
\alpha \\
\beta
\end{array}\right] \equiv M\left[\begin{array}{l}
1 \\
0
\end{array}\right] \equiv\left[\begin{array}{l}
0 \\
1
\end{array}\right](\bmod 2) \text {, } \\
& \text { if } n \equiv 1(\bmod 3) \text {, then }\left[\begin{array}{l}
\alpha \\
\beta
\end{array}\right] \equiv P\left[\begin{array}{l}
1 \\
0
\end{array}\right] \equiv\left[\begin{array}{l}
1 \\
1
\end{array}\right](\bmod 2), \\
& \text { if } n \equiv 0(\bmod 3) \text {, then }\left[\begin{array}{l}
\alpha \\
\beta
\end{array}\right] \equiv\left[\begin{array}{l}
1 \\
0
\end{array}\right](\bmod 2) .
\end{aligned}
$$

We deduce the following useful result

Proposition 3.11. Let $G \in \mathbf{G}$ and $\frac{\alpha}{\beta}=\left[e_{1}, \ldots, e_{n}\right]=P G P(\infty)$. Let $K=S\left(\frac{\alpha}{\beta}\right)$ and $N=\operatorname{cn}\left(\frac{\alpha}{\beta}\right)$. The following properties are equivalent:

1. $G$ is palindromic (i.e. $\bar{G}=G$ ).

2. the sequence of sign changes in $\left[e_{1}, \ldots, e_{n}\right]$ is palindromic (i.e. $\left.e_{i} e_{i+1}=e_{n-i} e_{n-i+1}\right)$.

3. $\beta^{2} \equiv(-1)^{N-1}(\bmod \alpha)$.

Furthermore we have

- $\beta^{2} \equiv-1(\bmod \alpha)$ (i.e. $K=\bar{K}$ is amphicheiral) if and only if $N$ is even and $G=\bar{G}$. Furthermore, the length $n=\ell\left(\frac{\alpha}{\beta}\right)$ is even and the sequence $\left[e_{1}, \ldots, e_{n}\right]$ is palindromic (i.e. $\left.e_{i}=e_{n-i+1}\right)$.

- $\beta^{2} \equiv 1(\bmod \alpha)$ if and only if $N$ is odd and $G=\bar{G}$ or $N$ is even and $\widehat{G}=\bar{G}$ (in this case $K$ is a 2-component link).

Proof. From Remark 3.9, we deduce that $G=\bar{G}$ is palindromic if and and only if the sequence of sign changes in $\left[e_{1}, \ldots, e_{n}\right]$ is palindromic.

Let $0<\beta^{\prime}<\alpha$ such that $\beta^{\prime} \beta \equiv(-1)^{N-1}(\bmod \alpha)$. We have from the previous proposition: $\frac{\alpha}{\beta^{\prime}}=P \bar{G} P(\infty)$. We thus deduce that $G=\bar{G}$ is equivalent to $\beta=\beta^{\prime}$, that is $\beta^{2} \equiv(-1)^{N-1}(\bmod \alpha)$.

Suppose now that $\beta^{2} \equiv 1(\bmod \alpha)$. If $N$ is even then $\beta^{\prime}=\alpha-\beta$, that is $P \bar{G} P=P \widehat{G} P$ and $\bar{G}=\widehat{G}$. We have $p+2 m=m+2 p-2$ and then $2 n=2(p+2 m)=3 N-2$. This implies $n \equiv 2(\bmod 3)$. By Lemma 3.10, $\alpha$ is even and $K$ is a two-component link. If $N$ is odd then $\beta^{\prime}=\beta$ and $G=\bar{G}$ by the first part of our proof.

Suppose now that $\beta^{2} \equiv-1(\bmod \alpha)$. If $N$ is odd then $\beta^{\prime}=\alpha-\beta$ and by the same argument we should have $n=3 N-n-2$, which would imply that $N$ is even. We deduce that amphicheiral rational links have even crossing numbers and from $\beta^{\prime}=\beta$ we get $G=\bar{G}$. The crossing number $N=m+p$ is even and $G$ is palindromic so $m$ and $p$ are both even. Consequently $n=p+2 m$ is even and the number of sign changes is even. We thus have $e_{n}=1$ and $\left(e_{n}, \ldots, e_{1}\right)=\left(e_{1}, \ldots, e_{n}\right)$, using remark 3.9. 


\section{Chebyshev diagrams of rational knots}

Definition 4.1. We say that a knot in $\mathbf{R}^{3} \subset S S^{3}$ has a Chebyshev diagram $\mathcal{C}(a, b)$, if a and $b$ are coprime and the Chebyshev curve

$$
\mathcal{C}(a, b): x=T_{a}(t) ; y=T_{b}(t)
$$

is the projection of some knot which is isotopic to $K$.

In [KP3] we proved that every knot has a Chebyshev diagram.

Proposition 4.2. Let $K$ be a knot, $b r(K)$ its bridge number. Let $m \geq b r(K)$ be an integer. Then $K$ has a projection which is a Chebyshev curve $\mathcal{C}(a, b): x=T_{a}(t) ; y=T_{b}(t)$, where $a=2 m-1$ and $b \equiv 2(\bmod 2 a)$.

This result is analogous to a theorem of Lamm for Lissajous curves (see [La2, BDHZ]).

In the case of two-bridge knots, we give an easy proof of this result. Moreover, we give an explicit method to get a minimal Chebyshev diagram $\mathcal{C}(3, b)$.

Theorem 4.3. Let $K$ be a two-bridge knot with crossing number $N$.

1. $K$ has a Chebyshev diagram $\mathcal{C}(3, b)$ with $N<b<\frac{3}{2} N$.

2. There exists $\frac{\alpha}{\beta}>1$ such that $K=S\left( \pm \frac{\alpha}{\beta}\right)$ and $\ell\left(\frac{\alpha}{\beta}\right)<\frac{3}{2} N-1$. If $\frac{\alpha}{\beta}$ is such a fraction, then $b=\ell\left(\frac{\alpha}{\beta}\right)+1$ is the minimal integer such that $K$ has a Chebyshev diagram $\mathcal{C}(3, b)$.

3. If $K$ has the Conway normal forms $C\left(\varepsilon_{1}, \ldots, \varepsilon_{n}\right), \varepsilon_{i}= \pm 1$, and $C\left(e_{1}, \ldots, e_{n}\right), e_{i}= \pm 1$ of minimal length, then we have either $\left(e_{1}, \ldots, e_{n}\right)=\left(\varepsilon_{1}, \ldots, \varepsilon_{n}\right)$ or $\left(e_{1}, \ldots, e_{n}\right)=$ $(-1)^{n+N}\left(\varepsilon_{n}, \ldots, \varepsilon_{1}\right)$.

Proof. Let $K$ be a two-bridge knot. Let $r=\frac{\alpha}{\beta}>1$ such that $K=S(r)$. We have $\bar{K}=S\left(r^{\prime}\right)$ where $r^{\prime}=\frac{\alpha}{\alpha-\beta}$. From Proposition 3.8 and Proposition 2.5, we have $\ell(r)+\ell\left(r^{\prime}\right)=3 N-2$ and therefore $N \leq \min \left(\ell(r), \ell\left(r^{\prime}\right)\right)<\frac{3}{2} N$. From Lemma 3.10, we have $\ell(r) \not \equiv 2(\bmod 3)$ so $\ell(r) \neq \ell\left(r^{\prime}\right)$ and $n=\min \left(\ell(r), \ell\left(r^{\prime}\right)\right)<\frac{3}{2} N-1$. Let us suppose now that $n=\ell(r)<\ell\left(r^{\prime}\right)$ and consider the 1-regular continued fraction expansion $r=\left[e_{1}, \ldots, e_{n}\right]$. Then $C\left(e_{1}, \ldots, e_{n}\right)$ is a Conway normal form for $K$. This Conway normal form corresponds to a Chebyshev $\operatorname{diagram} \mathcal{C}(3, n+1): x=T_{3}(t), y=T_{n+1}(t)$ and $b=n+1$. If $n=\ell\left(r^{\prime}\right)$ and $r^{\prime}=\left[e_{1}^{\prime}, \ldots, e_{n}^{\prime}\right]$, we would have considered the Conway normal form $C\left(-e_{1}^{\prime}, \ldots,-e_{n}^{\prime}\right)$ for $K=S\left(-r^{\prime}\right)$.

Let us consider $\gamma$ such that $\beta \gamma \equiv 1(\bmod \alpha)$ and $0<\gamma<\alpha$. Let $\rho=\frac{\alpha}{\gamma}$ and $\rho^{\prime}=\frac{\alpha}{\alpha-\gamma}$. We have $K=S(\rho)$ and $\bar{K}=S\left(\rho^{\prime}\right)$ and from Proposition 3.8, $n=\ell(r)=\ell(\rho)<\ell\left(\rho^{\prime}\right)=\ell\left(r^{\prime}\right)$.

Suppose that $K=C\left(\varepsilon_{1}, \ldots, \varepsilon_{\nu}\right), \varepsilon_{i}= \pm 1$. Let us show that $\nu \geq n$. We have $K=S(x)$ where $x=\left[\varepsilon_{1}, \ldots, \varepsilon_{\nu}\right]$ and $x=\frac{\alpha}{\beta+k \alpha}$ or $x=\frac{\alpha}{\gamma+l \alpha}$ where $k, l \in \mathbf{Z}$. We have $\ell(x)=\nu$ if $\left(\varepsilon_{1}, \ldots, \varepsilon_{\nu}\right)$ is 1-regular and $\nu \geq \ell(x)+3$ otherwise. 
- If $k=2 p>0$ then we have $x=(M P)^{p} r$ so $\ell(x)=\ell(r)+3 p>\ell(r)$.

- If $k=2 p+1>0$ then $x=(M P)^{p} M\left(\frac{1}{r}\right)$ so $\ell(x)=\ell(1 / r)+3 p+2=\ell\left(r^{\prime}\right)+3 p+3>\ell\left(r^{\prime}\right)$.

- If $k=-(2 p+1)<0$ then $-x=(M P)^{p}\left(r^{\prime}\right)$ so $\ell(x)=\ell(-x)=\ell\left(r^{\prime}\right)+3 p>\ell\left(r^{\prime}\right)$.

- If $k=-2 p>0$ then $-x=(M P)^{p-1} M\left(\frac{1}{r^{\prime}}\right)$ so $\ell(x)=\ell\left(1 / r^{\prime}\right)+3 p-1=\ell(r)+3 p>\ell(r)$.

If $x=\frac{\alpha}{\gamma+l \alpha}$, we obtain the same relations. We deduce that $\nu \geq \min \left(\ell(r), \ell\left(r^{\prime}\right)\right)=n$ and the second point. We deduce that $\nu=n$ if and only if $x=\frac{\alpha}{\beta}$ or $x=\frac{\alpha}{\gamma}$. In this case, we get the third point using Proposition 3.8 and the uniqueness of the 1-regular continued fraction expansion (Theorem 3.1).

\section{Algorithm 4.4 (Computing the minimal Chebyshev diagram).}

Let $K=S\left(\frac{\alpha}{\beta}\right), \frac{\alpha}{\beta}>1$. First compute the 1-regular sequence $\frac{\alpha}{\beta}=\left[e_{1}, \ldots, e_{n}\right], e_{i}=$ \pm 1 . $C\left(e_{1}, \ldots, e_{n}\right)$ is the Conway normal form corresponding to the Chebyshev diagram $\mathcal{C}(3, n+1)$.

If $n<\frac{3}{2} N-1$, then $b=n+1$ is the smallest integer such that has a Chebyshev diagram $x=T_{3}(t), y=T_{b}(t)$, from Proposition 4.3 .

If $n>\frac{3}{2} N-1$, let us consider the 1-regular continued fraction $\frac{\alpha}{\alpha-\beta}=\left[\varepsilon_{1}, \ldots, \varepsilon_{n^{\prime}}\right]$. We have $n^{\prime}<\frac{3}{2} N-1$, by Proposition 3.8. $C\left(-\varepsilon_{1}, \ldots,-\varepsilon_{n^{\prime}}\right)$ is the Conway normal form corresponding to the Chebyshev diagram $\mathcal{C}\left(3, n^{\prime}+1\right)$ of $K=S\left(-\frac{\alpha}{\alpha-\beta}\right)$. This last diagram is minimal by Proposition 4.3 .

Remark 4.5 (Minimality condition). First compute $G \in \mathbf{G}$ such that $\frac{\alpha}{\beta}=P G P(\infty)$. Let $p=\operatorname{deg}_{P}(P G P)$ and $m=\operatorname{deg}_{M}(P G P)$. By remark 3.6, we have $n=p+2 m$ and $N=p+m$. Consequently, the minimality condition $n<\frac{3}{2} N-1$ is equivalent to $p \geq m+3$.

Example 4.6 (Torus knots). The Schubert fraction of the torus knot $\mathrm{T}(2,2 k+1)$ is $2 k+1$. We have $P M(x)=x+2$, and then $(P M)^{k}(x)=x+2 k,(P M)^{k} P(x)=2 k+1+\frac{1}{x}$. So we get the continued fraction of length $3 k+1: 2 k+1=(P M)^{k} P(\infty)$. This shows that the torus knot $\mathrm{T}(2,2 k+1)$ has a Chebyshev diagram $\mathcal{C}(3,3 k+2)$. This is not a minimal diagram.

On the other hand, we get $(P M)^{k-1} P^{2}(\infty)=2 k$ so $\frac{2 k+1}{2 k}=P(P M)^{k-1} P^{2}(\infty)>1$. This shows that the torus knot $\mathrm{T}(2,2 k+1)$ has a Chebyshev diagram $\mathcal{C}(3,3 k+1)$. This diagram is minimal by Remark 4.5. We proved in KP3 that $\mathrm{T}(2,2 k+1)$ is in fact a harmonic knot parametrized by $x=T_{3}(t), y=T_{3 k+2}(t), z=T_{3 k+1}(t)$, that is $\operatorname{deg} y+\operatorname{deg} z=3(2 k+1)$. 
Example 4.7 (Twist knots). The twist knot $\mathcal{T}_{n}$ is defined by $\mathcal{T}_{n}=S\left(n+\frac{1}{2}\right)$.

From $P^{3}(x)=\frac{3 x+2}{2 x+1}$, we get the continued fraction of length $3 k+3: \frac{4 k+3}{2}=(P M)^{k} P^{3}(\infty)$. This shows that the twist knot $\mathcal{T}_{2 k+1}$ has a Chebyshev diagram $\mathcal{C}(3,3 k+4)$, which is minimal by Remark 4.5 .

We also deduce that $P(P M)^{k-1} P^{3}(\infty)=P\left(\frac{1}{2}(4 k-1)\right)=\frac{4 k+1}{4 k-1}$. This shows that the twist knot $\mathcal{T}_{2 k}$ has a minimal Chebyshev diagram $\mathcal{C}(3,3 k+2)$.

We shall see that these knots are not harmonic knots for $a=3$ and we will give explicit bounds for their polynomial parametrizations.

Example 4.8 (Generalized stevedore knots). The generalized stevedore knot $\mathcal{S}_{k}$ is defined by $\mathcal{S}_{k}=S\left(2 k+2+\frac{1}{2 k}\right)$. We have

$$
(M P)^{k}=\left[\begin{array}{cc}
1 & 0 \\
2 k & 1
\end{array}\right],(P M)^{k}=\left[\begin{array}{cc}
1 & 2 k \\
0 & 1
\end{array}\right]
$$

so $2 k+2+\frac{1}{2 k}=(P M)^{k+1}(M P)^{k}(\infty)$. This shows that the stevedore knot $\mathcal{S}_{k}$ has a Chebyshev diagram $\mathcal{C}(3,6 k+4)$. It is not minimal and we see, using Remark 4.5, that the knot $\mathcal{S}_{k}$ also has a minimal Chebyshev diagram $\mathcal{C}(3,6 k+2)$. Moreover, using Proposition 3.8, we get

$$
\frac{(k+1)^{2}}{(k+1)^{2}-2 k}=P^{2}(M P)^{k}(P M)^{k-1} P^{2}(\infty)
$$

\section{Polynomial parametrization of rational knots}

Definition 5.1. Let $\mathcal{D}(K)$ be a diagram of a knot having crossing points corresponding to the parameters $t_{1}, \ldots, t_{2 m}$. The Gauss sequence of $\mathcal{D}(K)$ is defined by $g_{k}=1$ if $t_{k}$ corresponds to an overpass, and $g_{k}=-1$ if $t_{k}$ corresponds to an underpass.

Theorem 5.2. Let $K$ be a two-bridge knot of crossing number $N$. Let $x=T_{3}(t), y=T_{b}(t)$ be the minimal Chebyshev diagram of $K$. Let $c$ denote the number of sign changes in the corresponding Gauss sequence. Then we have

$$
b+c=3 N .
$$

Proof. Let $s$ be the number of sign changes in the Conway normal form of $K$. By Proposition 2.5 we have $N=b-1-s$. From this we deduce that our condition is equivalent to $3 s+c=2 b-3$. Let us prove this assertion by induction on $s$. If $s=0$ then the diagram of $K$ is alternating, and we deduce $c=2(b-1)-1=2 b-3$.

Let $C\left(e_{1}, e_{2}, \ldots, e_{b-1}\right)$ be the Conway normal form of $K$. We may suppose $e_{1}=1$. We shall denote by $M_{1}, \ldots, M_{b-1}$ the crossing points of the diagram, and by $x_{1}<x_{2}<\cdots<$ 
$x_{b-1}$ their abscissae. Let $e_{k}$ be the first negative coefficient in this form. By the 1-regularity of the sequence we get $e_{k+1}<0$, and $3 \leq k \leq b-1$.

Let us consider the knot $K^{\prime}$ defined by its Conway normal form

$$
K^{\prime}=C\left(e_{1}, e_{2}, \ldots, e_{k-1},-e_{k},-e_{k+1}, \ldots,-e_{b-1}\right) .
$$

We see that the number of sign changes in the Conway sequence of $K^{\prime}$ is $s^{\prime}=s-1$. By induction, we get for the knot $K^{\prime}: 3 s^{\prime}+c^{\prime}=2 b-3$.

The plane curve $x=T_{3}(t), y=T_{b}(t)$ is the union of three arcs where $x(t)$ is monotonic. Let $\Gamma$ be one of these arcs. $\Gamma$ contains (at least) one point $M_{k}$ or $M_{k+1}$. Let $j$ be the first
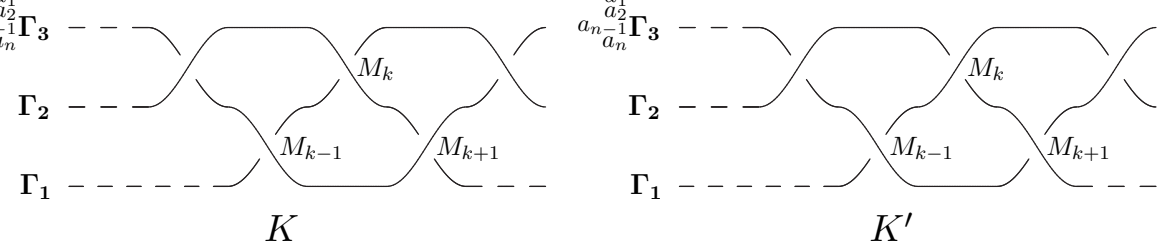

Figure 5: The modification of Gauss sequences

integer in $\{k, k+1\}$ such that $M_{j}$ is on $\Gamma$, and let $j_{-}<j$ be the greatest integer such that $M_{j_{-}} \in \Gamma$. In figure 5, we have for $\Gamma_{1}: j=k, j^{-}=k-1$, for $\Gamma_{2}: j=k, j^{-}=k-2$, for $\Gamma_{3}$ : $j=k+1, j^{-}=k-1$.

On each $\operatorname{arc} \Gamma$, there is a sign change in the Gauss sequence iff the corresponding Conway signs are equal. Then, since the Conway signs $s\left(M_{j_{-}}\right)$and $s\left(M_{j}\right)$ are different, we see that the corresponding Gauss signs are equal. Now, consider the modifications in the Gauss sequences when we transform $K$ into $K^{\prime}$. Since the the Conway signs $s\left(M_{h}\right), h \geq k$ are changed, we see that we get one more sign change on every arc $\Gamma$. Thus the number of sign changes in the Gauss sequence of $K^{\prime}$ is $c^{\prime}=c+3$. We get $3 s+c=3\left(s^{\prime}+1\right)+c^{\prime}-3=$ $3 s^{\prime}+c^{\prime}=2 b-3$, which completes our induction proof.

Corollary 5.3. Let $K$ be a 2-bridge knot with crossing number $N$. Then there exist $b, c$, $b+c=3 N$, and an polynomial $C$ of degree $c$ such that the knot $x=T_{3}(t), y=T_{b}(t), z=C(t)$ is isotopic to $K$.

If $K$ is amphicheiral, then $b$ is odd, and the polynomial $C(t)$ can be chosen odd.

Proof. Let $b=n+1$ be the smallest integer such that $K$ has a Chebyshev diagram $x=T_{3}(t), y=T_{b}(t)$. By our theorem 5.2, the Gauss sequence $\left(g\left(t_{1}\right), \ldots, g\left(t_{2 n}\right)\right)$ of this diagram has $c=3 N-b$ sign changes. We choose $C$ such that $C\left(t_{i}\right) g\left(t_{i}\right)>0$ and we can realize it by choosing the roots of $C$ in $] t_{i}, t_{i+1}\left[\right.$ when $g\left(t_{i}\right) g\left(t_{i+1}\right)<0$.

If $K$ is amphicheiral, then $b$ is odd and the Conway form is palindromic by Proposition 3.11. Then our Chebyshev diagram is symmetrical about the origin. We see that the Gauss sequence is odd: $g\left(t_{h}\right)=-g\left(-t_{h}\right)$. This implies that the polynomial $C(t)$ is odd when we choose its roots to be, for example, $\frac{1}{2}\left(t_{i}+t_{i+1}\right)$ where $g\left(t_{i}\right) g\left(t_{i+1}\right)<0$. 
Remark 5.4. When $K$ if amphicheiral, it can be parametrized by three odd polynomials. In this case the central symmetry $(x, y, z) \mapsto(-x,-y,-z)$ reverses the orientations of both $K$ and $S S^{3}$. This gives a simple proof of a famous theorem of Hartley and Kawauchi: every amphicheiral rational knot is strongly negative amphicheiral ([HK, $\mathrm{Kaw}$ ).

Theorem 5.2 provides an effective polynomial parametrization $(x(t), y(t), z(t)$ with $\operatorname{deg} x=$ $3, \operatorname{deg} y+\operatorname{deg} y=3 N$. We conjecture:

Conjecture 5.5. Let $K$ be a rational knot of crossing number $N$. Let $(x(t), y(t), z(t))$ be a polynomial parametrization of $K$ with $\operatorname{deg} x=3$, then we have $\operatorname{deg} y+\operatorname{deg} z \geq 3 N$.

We shall give several examples of polynomial parametrizations of rational knots with Chebyshev diagrams $\mathcal{C}(3, b)$.

\section{Parametrizations of the torus knots}

The torus knot $\mathrm{T}(2,2 n+1)=S(2 n+1)$ has a minimal Chebyshev diagram $\mathcal{C}(3,3 k+1)$. It can be parametrized by $z=C(t)$ where $\operatorname{deg}(C)=3 k+2$. Actually we proved in [KP3] that $C=T_{3 k+2}$ is convenient.

\section{Parametrizations of the twist knots}

The twist knot $\mathcal{T}_{m}=S\left(m+\frac{1}{2}\right)$ has crossing number $m+2$. We shall see that $\mathcal{T}_{m}$ is not a harmonic knot $\mathrm{H}(3, b, c)$ because $2^{2} \not \equiv \pm 1(\bmod 2 m+1)$ except when $m=2$ (the figure-eight knot) or $m=1$ (trefoil). From example 4.7, we know that:

- $\mathcal{T}_{2 k+1}$ can be parametrized by $x=T_{3}(t), y=T_{3 k+4}, z=C(t)$ where $\operatorname{deg}(C)=3 k+5$.

- $\mathcal{T}_{2 k}$ can be parametrized by $x=T_{3}(t), y=T_{3 k+2}, z=C(t)$ where $\operatorname{deg}(C)=3 k+4$.

Example 5.6 (The 3-twist knot). $\mathcal{T}_{3}$ is the 3 -twist knot $\overline{5}_{2}$. It is the harmonic knot $\mathrm{H}(4,5,7)$ (see [KP3]). It can also be parametrized by

$$
x=T_{3}(t), y=T_{7}(t), z=t(4 t+3)(3 t+1)(6 t-5)\left(12 t^{2}-11\right)\left(2 t^{2}-1\right)
$$

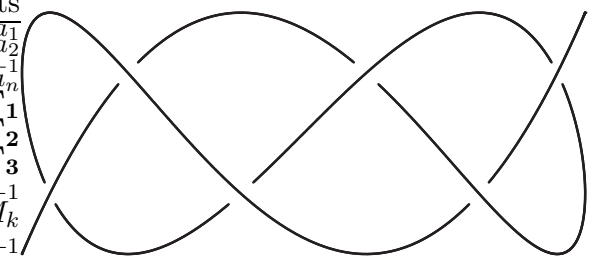

Figure 6: Diagram of the 3-twist knot $\overline{5}_{2}=S\left(\frac{7}{2}\right)$ 


\section{Parametrizations of the generalized stevedore knots}

The stevedore knot $\mathcal{S}_{m}=S\left(2 m+2+\frac{1}{2 m}\right)$ can be represented by $x=T_{3}(t), y=$ $T_{6 m+2}(t), z=C(t)$ where $C(t)$ is a polynomial of degree $6 m+4$.

Proof. This is a consequence of 4.8 and Corollary 5.3.

Example 5.7 (The knot $6_{1}=\mathcal{S}_{1}$ ). In the example 3.4, we get $\ell\left(\frac{9}{2}\right)=9, \ell\left(\frac{9}{7}\right)=7 . b=8$ is the minimal value for which $x=T_{3}(t), y=T_{8}(t)$ is a Chebyshev diagram for $\overline{6}_{1}$. The Gauss sequence associated to the Conway form $\overline{6}_{1}=C(-1,-1,-1,1,1,1,1)$ has exactly 10 sign changes. It is precisely

$$
[1,-1,-1,1,-1,1,-1,-1,1,-1,1,1,-1,1]
$$

We can check that

$$
x=T_{3}(t), y=T_{8}(t), z=(8 t+7)(5 t-4)\left(15 t^{2}-14\right)\left(2 t^{2}-1\right)\left(3 t^{2}-1\right)\left(15 t^{2}-1\right)
$$

is a parametrization of $\overline{6}_{1}$ of degree $(3,8,10)$. In [KP3] we gave the Chebyshev parametrization $6_{1}=\mathcal{C}\left(3,8,10, \frac{1}{100}\right)$. We shall see that $\overline{6}_{1}=S\left(\frac{9}{2}\right)$ is not a harmonic $\operatorname{knot} \mathrm{H}(3, b, c)$ because $2^{2} \not \equiv \pm 1(\bmod 9)$.

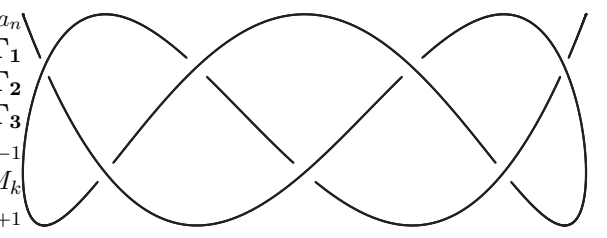

Figure 7: The knot 61

\section{The harmonic knots $\mathrm{H}(3, \mathrm{~b}, \mathrm{c})$}

In this paragraph we shall study Chebyshev knots with $\varphi=0$. Comstock (1897) found the number of crossing points of the harmonic curve parametrized by $x=T_{a}(t), y=T_{b}(t), z=$ $T_{c}(t)$. In particular, he proved that this curve is non-singular if and only if $a, b, c$ are pairwise coprime integers $([\mathrm{Com}])$. Such harmonic curves will be named harmonic $\operatorname{knots} \mathrm{H}(a, b, c)$ following the original denomination $([\mathrm{Com})$. These are not the harmonic knots defined by Trautwein $([\mathrm{Tr}]$ ), which are now referred to as Fourier knots (cf $[\mathrm{Cr}]$ ).

We shall need the following result proved in [KP3] 
Proposition 6.1. Let $a$ and $b$ be coprime integers. The $\frac{1}{2}(a-1)(b-1)$ double points of the Chebyshev curve $x=T_{a}(t), y=T_{b}(t)$ are obtained for the parameter pairs

$$
t=\cos \left(\frac{k}{a}+\frac{h}{b}\right) \pi, s=\cos \left(\frac{k}{a}-\frac{h}{b}\right) \pi,
$$

where $h, k$ are positive integers such that $\frac{k}{a}+\frac{h}{b}<1$.

Using the symmetries of Chebyshev polynomials, we see that this set of parameters is symmetrical about the origin. We shall need the following result proved in [KP3]. We will write $x \sim y$ when $\operatorname{sign}(x)=\operatorname{sign}(y)$.

Lemma 6.2. Let $\mathrm{H}(a, b, c)$ be the harmonic knot: $x=T_{a}(t), y=T_{b}(t), z=T_{c}(t) . A$ crossing point of parameter $t=\cos \left(\frac{k}{a}+\frac{h}{b}\right) \pi$, is a right twist if and only if

$$
D=(z(t)-z(s)) x^{\prime}(t) y^{\prime}(t)>0
$$

where

$$
z(t)-z(s)=T_{c}(t)-T_{c}(s)=-2 \sin \left(\frac{c h}{b} \pi\right) \sin \left(\frac{c k}{a} \pi\right)
$$

and

$$
x^{\prime}(t) y^{\prime}(t) \sim(-1)^{h+k} \sin \left(\frac{a h}{b} \pi\right) \sin \left(\frac{b k}{a} \pi\right) .
$$

From this lemma we immediately deduce

Corollary 6.3. Let $a, b, c$ be coprime integers. Suppose that the integer $c^{\prime}$ verifies $c^{\prime} \equiv$ $c(\bmod 2 a)$ and $c^{\prime} \equiv-c(\bmod 2 b)$. Then the knot $\mathrm{H}\left(a, b, c^{\prime}\right)$ is the mirror image of $\mathrm{H}(a, b, c)$.

Proof. At each crossing point we have $T_{c^{\prime}}(t)-T_{c^{\prime}}(s)=-\left(T_{c}(t)-T_{c}(s)\right)$.

Corollary 6.4. Let $a, b, c$ be coprime integers. Suppose that the integer $c$ is of the form $c=\lambda a+\mu b$ with $\lambda, \mu>0$. Then there exists $c^{\prime}<c$ such that $\mathrm{H}(a, b, c)=\overline{\mathrm{H}}\left(a, b, c^{\prime}\right)$

Proof. Let $c^{\prime}=|\lambda a-\mu b|$. The result follows immediately from corollary 6.3

In a recent paper, G. and J. Freudenburg have proved the following stronger result. There is a polynomial automorphism $\Phi$ of $\mathbf{R}^{3}$ such that $\Phi(\mathrm{H}(a, b, c))=\mathrm{H}\left(a, b, c^{\prime}\right)$. They also conjectured that the knots $\mathrm{H}(a, b, c), a<b<c, c \neq \lambda a+\mu b, \lambda, \mu>0$ are different knots ([FF], Conjecture 6.2).

When $a \leq 2$, it is easy to see that the harmonic knots $\mathrm{H}(a, b, c)$ are trivial knots.

The following result is the main step in the classification of the harmonic knots $\mathrm{H}(3, b, c)$. 
Theorem 6.5. Let $b=3 n+1, c=2 b-3 \lambda,(\lambda, b)=1$. The Schubert fraction of the knot $\mathrm{H}(3, b, c)$ is

$$
\frac{\alpha}{\beta}=\left[e_{1}, e_{2}, \ldots, e_{3 n}\right], \text { where } e_{k}=\operatorname{sign}(\sin k \theta) \text { and } \theta=\frac{\lambda}{b} \pi \text {. }
$$

If $0<\lambda<\frac{b}{2}$, its crossing number is $N=b-\lambda=\frac{b+c}{3}$, and we have $\beta^{2} \equiv \pm 1(\bmod \alpha)$.

Proof. Will be given in section [7, p. 21,

Corollary 6.6. The knots $\mathrm{H}(3, b, c)$ where $\frac{c}{2}<b<2 c, b \equiv 1(\bmod 3), c \equiv 2(\bmod 3)$ are different knots (even up to mirroring). Their crossing number is given by $b+c=3 N$.

Proof. Let $K=\mathrm{H}(3, b, c)$ and $\frac{\alpha}{\beta}>1$ be its 1-regular Schubert fraction given by Theorem 6.5. From Prop 4.3, $\min (b, c)$ is the minimum length of a Chebyshev diagram of $K$ and $\max (b, c)=3 N-\min (b, c)$. The pair $(b, c)$ is uniquely determined.

The following result gives the classification of harmonic knots $\mathrm{H}(3, b, c)$.

\section{Theorem 6.7.}

Let $K=\mathrm{H}(3, b, c)$. There exists a unique pair $\left(b^{\prime}, c^{\prime}\right)$ such that (up to mirror symmetry)

$$
K=\mathrm{H}\left(3, b^{\prime}, c^{\prime}\right), b^{\prime}<c^{\prime}<2 b^{\prime}, b^{\prime}+c^{\prime} \equiv 0(\bmod 3) .
$$

The crossing number of $K$ is $\frac{1}{3}\left(b^{\prime}+c^{\prime}\right)$, its fractions $\frac{\alpha}{\beta}$ are such that $\beta^{2} \equiv \pm 1(\bmod \alpha)$. Furthermore, there is an algorithm to find the pair $\left(b^{\prime}, c^{\prime}\right)$.

Proof. Let $K=\mathrm{H}(a, b, c)$ We will show that if the pair $(b, c)$ does not satisfy the condition of the theorem, then it is possible to reduce it.

If $c<b$ we consider $\mathrm{H}(3, c, b)=\overline{\mathrm{H}}(3, b, c)$.

If $b \equiv c(\bmod 3)$, we have $c=b+3 \mu, \mu>0$. Let $c^{\prime}=|b-3 \mu|$. We have $c^{\prime} \equiv \pm c(\bmod 2 b)$ and $c^{\prime} \equiv \mp c(\bmod 6)$. By Lemma 6.3, we see that $K=\overline{\mathrm{H}}\left(3, b, c^{\prime}\right)$ and we get a smaller pair.

If $b \not \equiv c(\bmod 3)$ and $c>2 b$, we have $c=2 b+3 \mu, \mu>0$. Let $c^{\prime}=|2 b-3 \mu|$. Similarly, we get $K=\overline{\mathrm{H}}\left(3, b, c^{\prime}\right)$. This completes the proof of existence. This uniqueness is a direct consequence of Corollary 6.6.

Remark 6.8. In $\left[\mathrm{FF}\right.$, it is proved (see Proposition 4.2) that $\mathrm{H}(3, b, c)$ and $\mathrm{H}\left(3, b^{\prime}, c^{\prime}\right)$ are algebraically equivalent.

Remark 6.9. Theorem 6.7 gives a positive answer to the Freudenburg conjecture for $a=3$.

Remark 6.10. Harmonic knots have Chebyshev parametrizations with the same degrees as the parametrizations we gave in section $\mathbf{5}$ (Theorem 5.2). 
Example 6.11. We get $\mathrm{H}(3,31,43)=\mathrm{H}(3,19,31)=\mathrm{H}(3,7,19)=\mathrm{H}(3,5,7)$. The crossing number of this knot is $4=\frac{1}{3}(5+7)$. With $b=5$ and $c=2 b-3$ we get the Conway normal form $C\left(\operatorname{sign}\left(\sin \frac{\pi}{5}\right), \operatorname{sign}\left(\sin \frac{2 \pi}{5}\right), \operatorname{sign}\left(\sin \frac{3 \pi}{5}\right), \operatorname{sign}\left(4 \sin \frac{\pi}{5}\right)\right)=C(1,1,1,1)$. Its Schubert fraction is $\frac{5}{3}=[1,1,1,1]$. It is the knot $4_{1}$.

\section{Examples}

As applications of Proposition [2.5, let us deduce the following results (already in [KP3]).

Corollary 6.12. The harmonic knot $\mathrm{H}(3,3 n+2,3 n+1)$ is the torus knot $\mathrm{T}(2,2 n+1)$.

Proof. The harmonic knot $K=\mathrm{H}(3,3 n+1,3 n+2)$ is obtained for $b=3 n+1, c=2 b-3 \lambda, \lambda=$ $n, \theta=\frac{n}{3 n+1} \pi$. If $j=1,2$, or 3 , and $k=0, \ldots, n-1$ we have $(3 k+j) \theta=k \pi+\frac{j k-n}{3 n+1}$, hence $\operatorname{sign}(\sin (3 k+j) \theta)=(-1)^{k}$, so that the Schubert fraction of $K$ is

$$
\left[1,1,1,-1,-1,-1, \ldots,(-1)^{n+1},(-1)^{n+1},(-1)^{n+1}\right]=\frac{2 n+1}{2 n} \approx-(2 n+1) .
$$

We see that $K$ is the mirror image of $\mathrm{T}(2,2 n+1)$, which completes the proof.

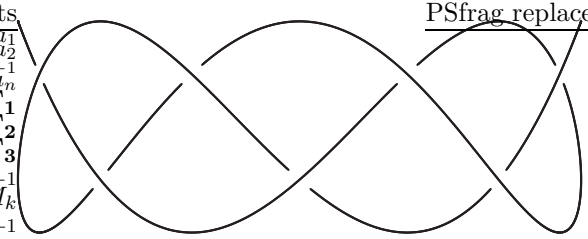

$\mathrm{H}(3,8,7)$

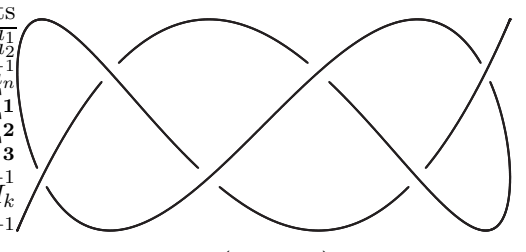

$\mathrm{H}(3,7,8)$

Figure 8: The torus knot $\mathrm{T}(2,5)=5_{1}$ and its mirror image

It is possible to parameterize the knot $\mathrm{T}(2,2 n+1)$ by polynomials of the same degrees and an alternating diagram ([KP2]). However, our Chebyshev parametrizations are easier to visualize. We conjecture that these degrees are minimal (see also [RS, KP1]).

Corollary 6.13. The harmonic knot $\mathrm{H}(3, b, 2 b-3)(b \not \equiv 0(\bmod 3))$ has crossing number $b-1$. The Chebyshev diagram of the projection on the $x y$-plane is alternating.

Proof. For this knot we have $\lambda=1, \theta=\frac{\pi}{b}$. The Conway normal form of the projection on the $x y$-plane is $(1,1, \ldots, 1)$. The Schubert fraction is the continued fraction of length $b-1$ : $[1,1, \ldots, 1]=\frac{F_{b}}{F_{b-1}}$ where $F_{n}$ are the Fibonacci numbers $\left(F_{0}=0, F_{1}=1, \ldots\right)$. 
Remark 6.14. J. C. Turner named these knots Fibonacci knots ([Tu]). In [KP3], we showed the more general result: the projection of $\mathrm{H}(a, b, a b-a-b)$ on the $x y$-plane is alternating. In [KP5], we have studied Fibonacci knots and generalized Fibonacci knots and showed that most of them are not Lissajous knots.

The two previous examples describe infinite families of harmonic knots. They have a Schubert fraction $\frac{\alpha}{\beta}$ with $\beta^{2} \equiv 1(\bmod \alpha)$ (torus knots) or with $\beta^{2} \equiv-1(\bmod \alpha)$ (Fibonacci knots with odd $b)$. There is also an infinite number of two-bridge knots with $\beta^{2}= \pm 1(\bmod \alpha)$ that are not harmonic.

Proposition 6.15. The knots (or links) $K_{n}=\mathcal{C}(1,1, \underbrace{-1, \ldots,-1}_{n+2}, 1,1), n>1$, are not harmonic knots $\mathrm{H}(3, b, c)$. Their crossing number is $n+4$ and their Schubert fraction $\frac{\alpha_{n}}{\beta_{n}}$ satisfy $\beta_{n}^{2} \equiv(-1)^{n+1}\left(\bmod \alpha_{n}\right)$.

Proof. We have $\frac{\alpha_{n}}{\beta_{n}}=P M P^{n} M P(\infty)$. Using the fact that $P^{n}=\left[\begin{array}{cc}F_{n+1} & F_{n} \\ F_{n} & F_{n-1}\end{array}\right]$ we deduce that

$$
P M P^{n} M P=\left[\begin{array}{cc}
5 F_{n+1} & F_{n+1}+F_{n-1} \\
F_{n+1}+F_{n-1} & F_{n-1}
\end{array}\right],
$$

that is $\alpha_{n}=5 F_{n+1}, \beta_{n}=F_{n+1}+F_{n-1}$. Taking determinants, we get $\beta_{n}^{2} \equiv(-1)^{n+1}\left(\bmod \alpha_{n}\right)$. Since $n+2 \geq 4$, it cannot be of the form $[\operatorname{sign}(\sin \theta), \operatorname{sign}(\sin 2 \theta), \ldots, \operatorname{sign}(\sin k \theta)]$.

If $n \equiv 2(\bmod 3), K_{n}$ is a two-component link.

If $n \equiv 1(\bmod 6)$ or $n \equiv 3(\bmod 6)$, we have $\beta_{n}^{2} \equiv 1\left(\bmod \alpha_{n}\right)$.

If $n \equiv 0(\bmod 6)$ or $n \equiv 4(\bmod 6), K_{n}$ is amphicheiral.

\section{Proof of theorem 6.5}

We study here the diagram of $\mathrm{H}(3, b, c)$ where $b=3 n+1$ and $c=2 b-3 \lambda$. The crossing points of the plane projection of $\mathrm{H}(3, b, c)$ are obtained for pairs of values $(t, s)$ where $t=\cos \left(\frac{m}{3 b} \pi\right), s=\cos \left(\frac{m^{\prime}}{3 b} \pi\right)$. For $k=0, \ldots, n-1$, let us consider

- $A_{k}$ obtained for $m=3 k+1, m^{\prime}=2 b-m$.

- $B_{k}$ obtained for $m=3 k+2, m^{\prime}=2 b+m$.

- $C_{k}$ obtained for $m=2 b-3 k-3, m^{\prime}=4 b-m$.

Then we have

- $x\left(A_{k}\right)=\cos \left(\frac{3 k+1}{b} \pi\right), y\left(A_{k}\right)=\frac{1}{2}(-1)^{k}$.

- $x\left(B_{k}\right)=\cos \left(\frac{3 k+2}{b} \pi\right), y\left(B_{k}\right)=\frac{1}{2}(-1)^{k+1}$. 


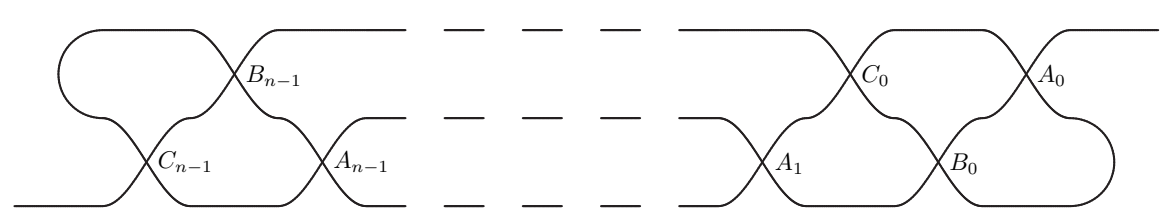

Figure 9: $\mathrm{H}(3,3 n+1, c), n$ even

- $x\left(C_{k}\right)=\cos \left(\frac{3 k+3}{b} \pi\right), y\left(C_{k}\right)=\frac{1}{2}(-1)^{k}$.

Hence our $3 n$ points satisfy

$$
x\left(A_{k-1}\right)>x\left(B_{k-1}\right)>x\left(C_{k-1}\right)>x\left(A_{k}\right)>x\left(B_{k}\right)>x\left(C_{k}\right), k=1, \ldots, n-1 .
$$

Using the identity $T_{a}^{\prime}(\cos \tau)=a \frac{\sin a \tau}{\sin \tau}$, we get $x^{\prime}(t) y^{\prime}(t) \sim \sin \left(\frac{m}{b} \pi\right) \sin \left(\frac{m}{3} \pi\right)$. We obtain

$$
\begin{aligned}
\text { for } A_{k}: \quad x^{\prime}(t) y^{\prime}(t) & \sim \sin \left(\frac{3 k+1}{b} \pi\right) \sin \left(\frac{3 k+1}{3} \pi\right) \sim(-1)^{k} . \\
\text { for } B_{k}: \quad x^{\prime}(t) y^{\prime}(t) & \sim \sin \left(\frac{3 k+2}{b} \pi\right) \sin \left(\frac{3 k+2}{3} \pi\right) \sim(-1)^{k} . \\
\text { for } C_{k}: \quad x^{\prime}(t) y^{\prime}(t) & \sim \sin \left(\frac{2 b-3 k-3}{b} \pi\right) \sin \left(\frac{2 b-3 k-3}{3} \pi\right) \\
& \sim-\sin \left(\frac{3 k+3}{b} \pi\right) \sin \left(-\frac{3 k+1}{3} \pi\right) \sim(-1)^{k} .
\end{aligned}
$$

The following identity will be useful in computing the sign of $z(t)-z(s)$.

$$
T_{c}(t)-T_{c}(s)=2 \sin \left(\frac{c}{6 b}\left(m^{\prime}-m\right) \pi\right) \sin \left(\frac{c}{6 b}\left(m+m^{\prime}\right) \pi\right) .
$$

We have, with $c=2 b-3 \lambda, \theta=\frac{\lambda}{b} \pi,($ and $b=3 n+1)$,

$$
\begin{aligned}
& \text { for } \begin{aligned}
& A_{k}: z(t)-z(s)=-2 \sin c \frac{\pi}{3} \sin \left(c \frac{m-b}{3 b} \pi\right) \text {. But } \\
& \qquad \sin c \frac{\pi}{3}=\sin \left(\frac{6 n+2-3 \lambda}{3} \pi\right)=(-1)^{\lambda} \sin \frac{2 \pi}{3},
\end{aligned}
\end{aligned}
$$

and

$$
\sin \left(c \frac{b-m}{3 b} \pi\right)=\sin \left(\left(2-\frac{3 \lambda}{b}\right) \frac{b-m}{3} \pi\right)=\sin \left(\frac{\lambda}{b}(m-b) \pi\right)=(-1)^{\lambda} \sin (3 k+1) \theta .
$$

We deduce that $z(t)-z(s) \sim \sin (3 k+1) \theta$. Finally, we obtain

$$
\operatorname{sign}\left(D\left(A_{k}\right)\right)=(-1)^{k} \operatorname{sign}(\sin (3 k+1) \theta) .
$$


- for $B_{k}: z(t)-z(s)=2 \sin c \frac{\pi}{3} \sin \left(\frac{c}{b} \cdot \frac{b+m}{3} \pi\right)$. We have

$$
\begin{aligned}
\sin \left(\frac{c}{b} \cdot \frac{b+m}{3} \pi\right) & =\sin \left(\left(2-\frac{3 \lambda}{b}\right) \frac{b+m}{3} \pi\right) \\
& =-\sin \left(\frac{\lambda}{b}(b+m) \pi\right)=(-1)^{\lambda+1} \sin (3 k+2) \theta .
\end{aligned}
$$

Then, using Equation 3, we get $z(t)-z(s) \sim-\sin (3 k+2) \theta$, and finally

$$
\operatorname{sign}\left(D\left(B_{k}\right)\right)=(-1)^{k+1} \operatorname{sign}(\sin (3 k+2) \theta) \text {. }
$$

- for $C_{k}: z(t)-z(s) \sim \sin \frac{2 c}{3} \pi \sin \left(\frac{c}{b}(k+1) \pi\right)$

We obtain

$$
\sim \sin \frac{4 \pi}{3} \sin \left(\left(2-\frac{3 \lambda}{b}\right)(k+1) \pi\right) \sim \sin (3 k+3) \theta .
$$

$$
\operatorname{sign}\left(D\left(C_{k}\right)\right)=(-1)^{k} \operatorname{sign}(\sin (3 k+3) \theta) .
$$

These results give the Conway normal form. If $n$ is odd, the Conway's signs of our points are

$$
\begin{aligned}
& s\left(A_{k}\right) \sim(-1)^{k} D\left(A_{k}\right) \sim \sin (3 k+1) \theta, \\
& s\left(B_{k}\right) \sim(-1)^{k+1} D\left(B_{k}\right) \sim \sin (3 k+2) \theta, \\
& s\left(C_{k}\right) \sim(-1)^{k} D\left(C_{k}\right) \sim \sin (3 k+3) \theta .
\end{aligned}
$$

In this case our result follows, since the fractions $\left[a_{1}, a_{2}, \ldots, a_{3 n}\right]$ and $(-1)^{3 n+1}\left[a_{3 n}, \ldots, a_{1}\right]$ define the same knot. If $n$ is even, the Conway's signs are the opposite signs, and we also get the Schubert fraction of our knot.

Since $0<\theta<\frac{\pi}{2}$, we see that there are not two consecutive sign changes in our sequence. We also see that the first two terms are of the same sign, and so are the last two terms. The Conway normal form is 1-regular and the total number of sign changes in this sequence is $\lambda-1$ : the crossing number of our knot is then $b-\lambda$. Finally, we get $\beta^{2} \equiv \pm 1$ by Proposition 3.11.

\section{Conclusion}

We have given here a complete classification of harmonic knots $\mathrm{H}(3, b, c)$ by computing explicitly their Schubert fraction. We have shown that when $b<c<2 b$ then $\mathrm{H}(3, b, c)$ has crossing number $N=\frac{1}{3}(b+c)$.

On the other hand we have shown that any rational knot of crossing number $N$ admits a polynomial parametrization of degrees $(a, b, c)$ where $a=3, N=\frac{1}{3}(b+c)$ and $N<b<c<$ $2 N$.

This is the first algorithm giving explicit polynomial parametrizations for the infinite family of rational knots. We also conjecture that these degrees are minimal $(a=3, b+c=3 N)$. 


\section{References}

[BZ] G. Burde, H. Zieschang, Knots, Walter de Gruyter, 2003

[BDHZ] A. Boocher, J. Daigle, J. Hoste, W. Zheng, Sampling Lissajous and Fourier knots, arXiv:0707.4210, (2007).

[BHJS] M. G. V. Bogle, J. E. Hearst, V. F .R. Jones, L. Stoilov, Lissajous knots, Journal of Knot Theory and its Ramifications, 3(2): 121-140, (1994).

[Com] E. H. Comstock, The Real Singularities of Harmonic Curves of three Frequencies, Trans. of the Wisconsin Academy of Sciences, Vol XI : 452-464, (1897).

[Con] J. H. Conway, An enumeration of knots and links, and some of their algebraic properties, Computational Problems in Abstract Algebra (Proc. Conf., Oxford, 1967), 329-358 Pergamon, Oxford (1970)

[Cr] P. R. Cromwell, Knots and links, Cambridge University Press, Cambridge, 2004. xviii+328 pp.

[Fi] G. Fischer, Plane Algebraic Curves, A.M.S. Student Mathematical Library Vol 15, 2001.

[FF] G. Freudenburg, J. Freudenburg, Curves defined by Chebyshev polynomials, 19 p., (2009), arXiv:0902.3440

[HK] R. Hartley, A. Kawauchi, Polynomials of amphicheiral knots, Math. Ann. 243 (1): 63-70 (1979)

[HZ] J. Hoste, L. Zirbel, Lissajous knots and knots with Lissajous projections, (2006), arXiv:math/0605632. To appear in Kobe Journal of mathematics, vol 24, no 2

[JP] V. F. R. Jones, J. Przytycki, Lissajous knots and billiard knots, Banach Center Publications, 42:145-163, (1998).

[Kaw] A. Kawauchi, editor, A Survey of Knot Theory, Birhäuser, 1996.

[KP1] P. -V. Koseleff, D. Pecker, On polynomial Torus Knots, Journal of Knot Theory and its Ramifications, Vol. 17 (12) (2008), 1525-1537.

[KP2] P. -V. Koseleff, D. Pecker, A construction of polynomial torus knots, to appear in Journal of AAECC, arXiv:0712.2408.

[KP3] P. -V. Koseleff, D. Pecker, Chebyshev knots, (2008), arXiv:0812.1089,

[KPR] P. -V. Koseleff, D. Pecker, F. Rouillier, The first rational Chebyshev knots, Conference MEGA 2009, Barcelona. 
[KP4] P. -V. Koseleff, D. Pecker, Chebyshev diagrams for rational knots, (2008), arXiv:0906.4083.

[KP5] P. -V. Koseleff, D. Pecker, On Fibonacci knots, (2009), arXiv:0908.0153.

[La1] C. Lamm, There are infinitely many Lissajous knots, Manuscripta Math., 93: 29-37, (1997).

[La2] C. Lamm, Cylinder knots and symmetric unions (Zylinder-knoten und symmetrische Vereinigungen), Ph.D. Thesis, Bonner Mathematische Schriften 321, Bonn, 1999.

[Mu] K. Murasugi, Knot Theory and its Applications, Boston, Birkhäuser, 341p., 1996.

[P1] D. Pecker, Simple constructions of algebraic curves with nodes, Compositio Math. 87 (1993), no. 1, 1-4.

[RS] A. Ranjan and R. Shukla, On polynomial representation of torus knots, Journal of knot theory and its ramifications, Vol. 5 (2) (1996) 279-294.

[Sh] A.R. Shastri, Polynomial representation of knots, Tôhoku Math. J. 44 (1992), $11-17$.

[St] A. Stoimenow, Generating functions, Fibonacci numbers and rational knots, J. Algebra 310(2) (2007), 491-525.

[Tr] A. Trautwein, Harmonic knots, Ph.D. Thesis, University of Iowa, 1994

[Tu] J.C. Turner, On a class of knots with Fibonacci invariant numbers, Fibonacci Quart. $24(1986), n^{\circ} 1,61-66$.

[Va] V. A. Vassiliev, Cohomology of knot spaces, Theory of singularities and its Applications, Advances Soviet Maths Vol 1 (1990) 